\title{
Association of Increased Red Cell Distribution Width with Malignant Transformation of Oral Submucous Fibrosis
}

\author{
Jaya Joshi ${ }^{1} \quad$ Kundendu Arya Bishen ${ }^{1}$ Sonam Gehi ${ }^{2}$ \\ Puneet Gupta ${ }^{4}$

\footnotetext{
${ }^{1}$ Department of Oral Pathology and Microbiology, Government College of Dentistry, Indore, Madhya Pradesh, India

${ }^{2}$ Department of Prosthodontics, Government College of Dentistry, Indore, Madhya Pradesh, India

${ }^{3}$ Shri Vaishnav Vidyapeeth Vishwavidyalaya, Indore,

Madhya Pradesh, India

${ }^{4}$ Department of Community Dentistry, Government College of

Dentistry, Indore, Madhya Pradesh, India
}

Pratiksha Kumar ${ }^{1}$ Anand Krishna Singh ${ }^{3}$

\author{
Address for correspondence Kundendu Arya Bishen, MDS, PhD, \\ Department of Oral and Maxillofacial Pathology, Government \\ College of Dentistry, Indore 452001, Madhya Pradesh, India \\ (e-mail: kundenduarya@gmail.com).
}

\begin{abstract}
Introduction Oral submucous fibrosis (OSMF) is associated with nutritional deficiencies-primarily of iron and vitamins and usually seem to be associated with anemia. Red cell distribution width (RDW) is one of the routinely assessed parameters in complete blood picture analysis of any patient. It measures range of variation in erythrocyte size. A high RDW value has been associated to adverse outcomes in several diseases and risk of death. However, the relationship of RDW and OSMF is yet to be established. Aim To analyze the association of RDW levels and other erythrocytic indices with clinical staging and prognosis of OSMF patients.

Materials and Methods Analysis of clinical stage and hematologic status of 86 patients comprising the study group (OSMF cases and OSMF with malignant changes) and control group was performed. It was done using Kruskal-Wallis analysis of variance test.

Results The values of mean corpuscular volume, mean corpuscular hemoglobin, and mean corpuscular hemoglobin concentration were below normal from the onset of oral submucous fibrosis and they continued to decrease as the disease progressed. On

Keywords

- blood indices

- oral submucous fibrosis

- red cell distribution width other hand, RDW values were found to be in normal range in initial stages of disease and they became high in stage IV OSMF and increased further in OSMF patients with malignant changes.

Conclusion We observed that higher values of RDW are strongly and independently associated with OSMF and OSCC, suggesting that RDW may contribute as most economic novel biochemical marker for progression and malignant transformation of OSMF.
\end{abstract}

\section{Introduction}

Red cell distribution width (RDW) is a parameter which is routinely evaluated along with complete blood count (CBC) test. RDW indicates anisocytosis in blood cells, that is, it measures heterogeneity in erythrocyte size and is helpful to clinically differentiate different types of anemia. Various studies have recently reported correlation between increased RDW levels and high mortality in several diseases in general population..$^{1,2}$ The pathogenesis behind increased RDW with disease progression and associated mortality has not yet been elucidated. It is assumed that chronic inflammation and poor nutritional status (e.g., iron, vitamin B12, and folate deficiency) provoke high levels of RDW via changes in erythropoiesis..$^{1,3,4}$
C2019 Bhojia Dental College and Hospital affiliated to Himachal Pradesh University
License terms

() (1) $\Theta \circledast$ 
Oral submucous fibrosis (OSMF) is a precancerous condition which is affecting $0.5 \%$ of Indian population. There has been constant rise in OSMF in India due to increased uptake of habit of consuming pan masala/gutkha due to effective marketing, fashion trend, and easy access. ${ }^{5}$ After areca nut, nutritional deficiencies such as that of iron and vitamins are considered as important etiologic factors behind OSMF. OSMF is regarded as Asian version of sideropenic dysphagia in which the chronic iron deficiency disrupts the integrity of epithelia of entire digestive tract making the mucosa more susceptible to various irritants. ${ }^{6}$ Several studies have pointed out significant hematological abnormalities in OSMF disease due to nutritional deficiency. ${ }^{7}$

Premaligmant tumors (e.g., OSMF) and malignant tumors evoke chronic inflammation and compromised nutritional status which account for patient's poor quality of life and they are obstacles in the treatment. Generally the age and stage/ grade of tumor (clinical and histological) are considered to risk-stratify patients and plan the therapy. Although these parameters provide useful information but the clinical conditions of OSMF patients-as the disease as well as treatment progresses are so complex that it becomes difficult to foretell exact prognosis. Biomarkers are valuable adjuncts for cancer diagnosis and treatment and finding accurate biomarkers defining the patient's general condition is still a significant challenge. As RDW levels reflect chronic inflammation and nutritional status, it might be associated with cancer progression. The present study was designed to explore the changes in profile of blood indices in OSMF cases and also to test the utility of RDW in authenticating the prognostic work up of patients of OSMF.

\section{Materials and Methods}

The study was approved by the institutional ethical committee. A sample population of 86 patients were recruited for study from the outpatients reporting in the Department of Oral Pathology and Microbiology of the college. The study sample comprised 56 patients. It included 43 patients clinically diagnosed and histologically confirmed for OSMF with no other systemic disease and having harmful habit of chewing gutkha/pan masala and 13 cases diagnosed of OSMF with oral squamous cell carcinoma (OSCC) along with harmful chewing habits. A control group of 30 healthy individuals whose age and sex were matched was taken. The OSMF patients were divided clinically into Grade I to IV as per criteria laid down by Andrade and Khanna. ${ }^{8}$ All OSMF patients with any systemic disease or with history of previous treatment for OSMF/OSCC or with any other hematological abnormalities were excluded from study.

The subjects were asked to complete a basic medical and dental questionnaire to obtain a meticulous history and other relevant information about OSMF. All subjects were requested to sign an attached consent form after being explained about the study in detail along with its purpose.

After thorough clinical evaluation, all the patients were subjected to hematological investigations with Automated
Cell Counter. The findings comprised RDW, mean corpuscular volume (MCV), mean corpuscular hemoglobin (MCH), mean corpuscular hemoglobin concentration (MCHC), hemoglobin, and granulocytes; $5 \mathrm{~mL}$ of peripheral blood was taken by standard venipuncture from ante cubital fossa of all patients and controls.

Following this, biopsy was performed for histologic confirmation of diagnosis. The histopathological evaluation of biopsy sample taken from representative site was done by the oral pathologist and reported accordingly. Healthy individuals without any underlying systemic or hematological diseases, subjected for hematological investigations for minor surgical procedures were taken as controls.

The data collected were transferred into electronic format and master table was prepared using Microsoft office 97-2003 Excel worksheet. For data analysis, IBMSPSS Statistics for Windows Version 24 was used. The obtained values were subjected to statistical evaluation. Mean and standard deviation of blood indices was calculated separately for each group and represented in a tabular form. Normality of the data was used using Shapiro-Wilk test. The data were found to not follow normal distribution. Hence non parametric tests were used. Difference in mean values between different stages was compared using Kruskal-Wallis analysis of variance. The $\alpha$ value was set at $5 \%$ and $p$ value less than 0.05 was considered to be statistically significant.

\section{Results}

The $\mathrm{Hb} \%$ continuously decreased as the OSMF progressed and was lowest in OSCC patients. MCV, MCH, and MCHC values were found to be below normal range from the onset of OSMF (stage 1 OSMF) and they continued to decrease as the disease progressed to higher stages and OSCC ( - Table 1 ).

On other hand RDW values kept on increasing as the disease progressed. Although there was an increasing trend, the values of RDW were found to be within normal range in initial and moderate stages of disease and it became high and crossed the normal value range in stage IV OSMF and increased further in patients of OSMF with malignant changes (-Fig. 1).

\section{Discussion}

OSMF is a well-known precancerous condition affecting the oral mucosa. It is considered to be a chronic progressive disorder and its clinical appearance depends on the stage at which it is detected. Intolerance to spicy food, burning sensation, and vesiculation are common complains of the majority of patients in initial stages. This follows complain of rigidity of lip, tongue, and palate causing limitation of mouth opening and tongue movement to varying degrees. Submucosal fibrosis affects most parts of the oral cavity, pharynx, and upper third of the esophagus. ${ }^{5}$

Most of the patients with OSMF have a definite habit history of areca nut chewing. Stage of the disease shows a 
Table 1 Table showing levels of hemoglobin, MCV, MCH, MCHC, and RDW in normal and OSMF patients

\begin{tabular}{|l|l|l|l|l|l|l|l|l|l|l|}
\hline \multirow{2}{*}{} & \multicolumn{2}{|c|}{ Hemoglobin } & \multicolumn{2}{c|}{ MCV } & \multicolumn{2}{c|}{ MCH } & \multicolumn{2}{c|}{ MCHC } & \multicolumn{2}{c|}{ RDW } \\
\cline { 2 - 11 } & Mean & SD & Mean & SD & Mean & SD & Mean & SD & Mean & SD \\
\hline Control & 13.43 & 1.46 & 86.28 & 6.56 & 28.64 & 1.78 & 33.24 & 0.64 & 13.76 & 0.01 \\
\hline OSMF grade 1 & 14.49 & 1.74 & 96.00 & 5.34 & 27.46 & 1.78 & 32.43 & 0.36 & 14.18 & 0.01 \\
\hline OSMF grade 2 & 13.61 & 1.52 & 89.52 & 11.24 & 24.34 & 3.03 & 30.63 & 2.35 & 14.40 & 0.01 \\
\hline OSMF grade 3 & 12.81 & 1.96 & 84.98 & 9.04 & 23.55 & 4.54 & 26.14 & 2.64 & 14.38 & 0.01 \\
\hline OSMF grade 4 & 11.99 & 1.46 & 82.57 & 9.80 & 21.58 & 3.48 & 23.83 & 2.03 & 15.44 & 0.01 \\
\hline $\begin{array}{l}\text { OSMF with malignant } \\
\text { changes }\end{array}$ & 10.62 & 1.38 & 86.16 & 6.78 & 22.17 & 2.57 & 21.00 & 1.70 & 16.20 & 0.02 \\
\hline $\begin{array}{l}\text { Kruskal-Wallis analysis of } \\
\text { variance }\end{array}$ & $p<0.001$ & Sig & $p<0.001$ & Sig & $p<0.001$ & Sig & $p<0.001$ & Sig & $p<0.001$ & Sig \\
\hline
\end{tabular}

Abbreviations: $\mathrm{MCH}$, mean corpuscular hemoglobin; $\mathrm{MCHC}$, mean corpuscular hemoglobin concentration; MCV, mean corpuscular volume; OSMF, oral submucous fibrosis; RDW, red cell distribution width.

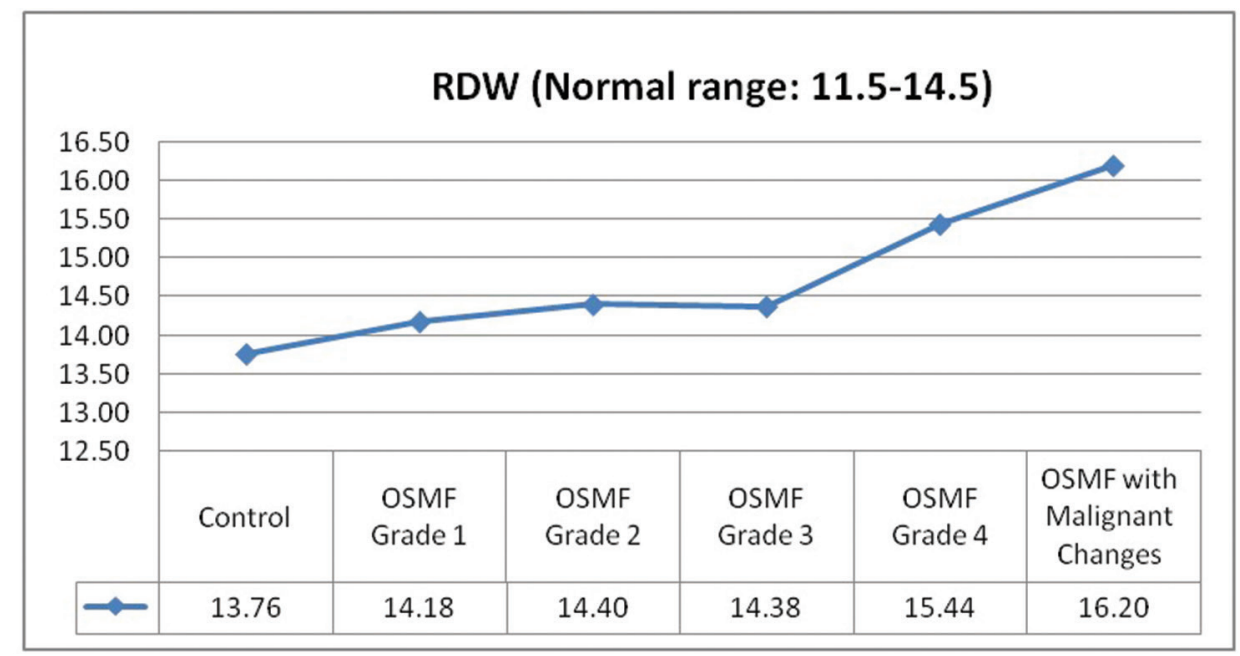

Fig. 1 Graph showing comparison of red cell distribution width levels in control group, different grades of OSMF and OSMF with malignant changes. OSMF, oral submucous fibrosis.

clear dose dependent relationship with both frequency and duration of areca nut chewing habit. ${ }^{9}$ Nutritional deficiencies (primarily of iron and vitamins), immunologic processes, and genetic predisposition are also implicated in etiology of OSMF. ${ }^{10}$ The clinical manifestations of OSMF render a phase for difficulty in consumption of the normal diet by patient who then manipulate the dietary/food habits and decrease the overall intake of conventional food or diet, thus leading to malnutrition and deficiency. ${ }^{11}$

Deficiency of iron, vitamin $B_{12}$, folate, and other trace elements due to nutritional depletion cause iron deficiency anemia affect entire oral mucosa its manifestations can be summarized as generalized oral mucosal atrophy, angular cheilitis, candidal infections, atrophic glossitis, pallor, and stomatitis. ${ }^{12}$ Anemia initiates an altered cell-mediated immunity, which acts as a promoting factor for pathologic response of the lamina propria in OSMF. After a frank establishment of the lesion, anemia further perpetuates due to inadequate intake of food due to fibrosis and trismus. ${ }^{6}$

Hemoglobin levels and serum iron levels are regarded as important biochemical indicators for nutritional assessment.
Various authors have reported significant hematological abnormalities in OMSF. The changes in blood parameters reported include an increased blood sedimentation rate, increased eosinophil count, decreased $\mathrm{Hb} \%$, a decrease in serum iron level, and an increase in total iron binding capacity (TIBC). The assessment of blood indices in individuals with OSMF reveal normal hematocrit value, decreased MCV, MCH, and MCHC. The RDW value increases as disease progresses. ${ }^{5,7,13}$

Homeostasis of iron is a unique process because the level of iron in serum is maintained within normal range only by regulation of the absorption mechanism of iron through intestine and never excretion. ${ }^{12}$ This means that if iron deficiency sets in, it can be corrected only by oral iron absorption through duodenal mucosa which allows uptake of iron in appropriate quantities to balance exactly the required small amount of daily iron loss.

If this oral iron is not available due to manipulated/ decreased dietary/food habits of patient, for example, in OSMF, or iron losses are amplified by some disease process or if dietary iron is there but its absorption is impaired, a negative iron balance will result. The body counterbalances, the 
effects of this negative balance for a short duration by mobilization of iron from body stores. This result in depletion of tissue iron and the serum iron falls leading to failure of iron supply to the bone marrow affecting hemopoiesis which further aggravates the condition. ${ }^{14}$

Other than compromised dietary habits due clinical symptoms in OSMF patients, decreased iron levels in OSMF patients might be attributed to utilization of iron in collagen synthesis. Pathogenesis of OSMF involves derangement of collagen metabolism. Proline, an amino acid found collagen is incorporated in collagen in its hydroxylated form. Ferrous ion and ascorbic acid are consumed to bring about this hydroxylation reaction for collagen synthesis. In OSMF there is excess production of collagen and thus utilization of iron for the hydroxylation of proline and lysine results in decreased serum iron level. ${ }^{15}$

The RDW is reported routinely as part complete blood count $(\mathrm{CBC})$ using automated blood counts in laboratories. It reflects variability in the size, that is, anisocytosis in circulating red blood cells. It is utilized along with other blood indices (e.g., $\mathrm{MCV}, \mathrm{MCH}$, and $\mathrm{MCHC}$ ) to diagnose various anemias confirmatively, especially those that are microcytic hypochromic type caused due to iron deficiency and vitamin $B_{12}$ or folic acid deficiencies. ${ }^{16}$

Due the fact that RDW is always available to clinicians as a part of routine $C B C$ investigation and it incurs no additional cost to patient, the diagnostic value of RDW should be explored. RDW has been reported recently to be a strong and independent predictor of adverse outcomes in many diseases. Various studies have shown correlation between increased RDW levels and high mortality rate in several diseases in general population. ${ }^{1,2}$ Thus it is prudent not to underestimate RDW changes in comparison to other novel prognostic markers of any disease.

We observed a graded and independent correlation between RDW and OSMF in the present study. The precise physiological mechanisms behind association of RDW with OSMF are still not well understood. It is possible that systemic factors such as inflammation and oxidative stress alter erythrocyte homeostasis, which may play a role in the association of RDW with disease progression in OSMF.

Inflammation may lead to an increased RDW by impairing iron metabolism and also by inhibiting the production of or response to erythropoietin. ${ }^{3,17}$ The oxidative stress may also contribute significantly to anisocytosis. Although erythrocytes have remarkable antioxidant capacity and they serve as primary "oxidative sink," they are susceptible to oxidative damage which reduces their survival leading to higher RDW values. ${ }^{18}$

Inflammation and lack of iron in the tissues lead to formation of improper vascular channels. This results in decreased vascularity and increased oxidative stress in fibrosed tissue. The decreased vascularity seen in advanced stages of OSMF leads to transient ischemia/hypoxia that stimulates release of certain growth factors such as hypoxia inducible factor (HIF). The hypoxic microenvironment seen in advanced OSMF brings about cellular adaptations such as reflex angiogenesis, neovascularization, and increased glycolysis-all of which are correlated with tumor invasion and metastasis. ${ }^{19}$

Although iron deficiency anemia and OSMF are different conditions, they both have similar clinical findings (e.g., blanching, dysphagia) and both cause similar effect on histology of mucosa (i.e., both cause atrophy) making it prone to malignant change. They both are inter related and create a vicious cycle aggravating one another. In both conditions, anisocytosis increases with disease progression and so the RDW values also increase as disease aggravates.

In present study also the RDW values kept on increasing with disease progression and it crossed the normal range in cases of highest grade OSMF and in OSMF with malignant changes. Thus, we conclude that high RDW values in grade IV OSMF patients can be considered as a definite hematologic marker suggestive for malignant transformation and poor prognosis.

The limitation of this study is that sample size was small and, therefore, the mechanisms that bring about the association of RDW with OSMF still remain ambiguous. They can be determined by performing large-scale epidemiological studies, which would clearly demonstrate the association between OSMF, its malignant transformation, and measure of anisocytosis (RDW). Also, it still remains to be determined, whether RDW is a simple marker of disease progression and malignant transformation, or a specific marker of high inflammation and iron deficiency which in turn mediate malignant transformation in OSMF.

\section{Conclusion}

In present study we found that high RDW values in grade IV OSMF patients can be considered as a definite hematologic marker suggestive for poor prognosis and malignant transformation in OSMF. As RDW values are always available as a part of routine $C B C$ investigation and it incurs no additional cost to patient, any change in its values must be analyzed as an independent predictor of adverse outcomes in OSMF.

\section{Funding}

None.

\section{Conflict of Interest}

None declared.

\section{References}

1 Patel KV, Ferrucci L, Ershler WB, Longo DL, Guralnik JM. Red blood cell distribution width and the risk of death in middle-aged and older adults. Arch Intern Med 2009;169(5):515-523

2 Patel KV, Semba RD, Ferrucci L, et al. Red cell distribution width and mortality in older adults: a meta-analysis. J Gerontol A Biol Sci Med Sci 2010;65(3):258-265

3 Douglas SW, Adamson JW. The anemia of chronic disorders: studies of marrow regulation and iron metabolism. Blood 1975;45(1):55-65

4 Ferrucci L, Guralnik JM, Woodman RC, et al. Proinflammatory state and circulating erythropoietin in persons with and without anemia. Am J Med 2005;118(11):1288 
5 Hegde K, Gharote H, Nair P, et al. Iron deficiency in oral submucous fibrosis: accelerator or a promoter? Int J Oral Maxillofac Pathol 2012;3(1):2-7

6 Rajendran R, Vijayakumar T, Vasudevan DM. An alternative pathogenetic pathway for oral submucous fibrosis (OSMF). Med Hypotheses 1989;30(1):35-37

7 Anuradha CD, Shamaladevi CS. Studies on the hematologic profile and trace elements in oral submucous fibrosis. J Clin Biochem Nutr 1995;19(1):9-17

8 Khanna JN, Andrade NN. Oral submucous fibrosis: a new concept in surgical management. Report of 100 cases. Int J Oral Maxillofac Surg 1995;24(6):433-439

9 Auluck A, Rosin MP, Zhang L, Sumanth KN. Oral submucous fibrosis, a clinically benign but potentially malignant disease: report of 3 cases and review of the literature. J Can Dent Assoc 2008;74(8):735-740

10 Cox SC, Walker DM. Oral submucous fibrosis. A review. Aust Dent J 1996;41(5):294-299

11 Anuraag AS, Puranik RS, Shreenivas SV, Shivakumar MS, Mahadevi B. The profile of red cell distribution width and some hematological parameters in patients of oral submucous fibrosis. J Oral Maxillofac Pathol 2014;18:S6

12 Bhattacharya PT, Misra SR, Hussain M. Nutritional aspects of essential trace elements in oral health and disease: an extensive review. Scientifica (Cairo) 2016;2016:5464373
13 Narang D, Rathod V, Shishodiya S, et al. Role of haematological profile in oral sub mucous fibrosis: a clinical study. J Adv Med Dent Sci Res 2015;3(4):6-8

14 Rennie JS, MacDonald DG, Dagg JH. Iron and the oral epithelium: a review. J R Soc Med 1984;77(7):602-607

15 Khanna SS, Karjodkar FR. Circulating immune complexes and trace elements (copper, iron and selenium) as markers in oral precancer and cancer : a randomised, controlled clinical trial. Head Face Med 2006;2:33

16 Aulakh R, Sohi I, Singh T, Kakkar N. Red cell distribution width (RDW) in the diagnosis of iron deficiency with microcytic hypochromic anemia. Indian J Pediatr 2009;76(3):265-268

17 Weiss G, Goodnough LT. Anemia of chronic disease. N Engl J Med 2005;352(10):1011-1023

18 Kiefer CR, Snyder LM. Oxidation and erythrocyte senescence. Curr Opin Hematol 2000;7(2):113-116

19 Chaudhary M, Bajaj S, Bohra S, Swastika N, Hande A. The domino effect: role of hypoxia in malignant transformation of oral submucous fibrosis. J Oral Maxillofac Pathol 2015;19(2):122-127 